\title{
Spectral Analysis of the Anisotropic Neutron Transport Kernel in Slab Geometry with Applications*
}

\author{
A. Leonard and T. W. Mulurkin \\ The RAND Corporation, Santa Monica, California
}

(Received 15 October 1963)

\begin{abstract}
A spectral analysis of the transport kernel for anisotropic scattering in finite slabs is achieved by first solving a type of generalized scattering problem for a subcritical slab. Initially, the scattering problem is stated as an inhomogeneous integral transport equation with a complex-valued source function. This is readily transformed to singular integral equations and linear constraints in which the space and angle variables enter as parameters. Dual singular equations appear in applications of Case's method to transport problems, but we cannot yet completely explain this duality. The singular equations are transformed to Fredholm equations by an extension of Muskhelishvili's standard method and by analytic continuation. It is shown that, for a wide class of scattering functions, this particular Fredholm reduction yields equations which converge rapidly under iteration for all neutron productions and slab thicknesses. The ultimate solution of the singular equations contains arbitrary constants which, when evaluated by the aforementioned linear constraints, display explicitly the Fredholm determinant and the eigenfunctions of the transport kernel. An immediate consequence of this result is the criticality condition and the associated neutron distribution. Specific applications to linear anisotropic and isotropic scattering in slab geometry are discussed. In addition, it is seen that the case of isotropic scattering in spheres can be treated with this method, and, in fact, the spectral analysis of the kernel for the slab problem immediately applies to the sphere kernel.
\end{abstract}

\section{INTRODUCTION}

$\mathbf{I}^{\mathrm{r}}$ $\mathrm{N}$ this paper, we consider an integral operator $\Lambda$, derivable from the steady-state transport equation for anisotropic scattering in a one-dimensional slab of finite thickness. By means of the theory of singular integral equations and analytic continuation, we reduce a certain scattering problem to a form suitable for computation. From this solution we obtain both the eigenvalues and eigenfunctions of the operator $\Lambda$. For isotropic scattering, these results give, by a familiar observation, ${ }^{1}$ a spectral analysis of the isotropic transport kernel for a finite sphere.

The spectral analysis is important in the determination of critical dimensions of nuclear reactors in which the energy of neutrons is considered constant. By the standard method of taking a Laplace transform in the time variable, ${ }^{1}$ these results can also be applied to determine the discrete spectrum of the time-dependent transport equation.

Recently, various transport problems for plane geometry have been treated by Case's singular eigenfunction method. ${ }^{2-5}$ This method is one of

* This research is sponsored by the United States Air Force under Project RAND-Contract No. AF 49(638)-700 monitored by the Directorate of Development Planning, Deputy Chief of Staff, Research and Development Headquarters, United States Air Force.

1 B. Davison, Neutron Transport Theory (Oxford University Press, London, 1957).

2 K. M. Case, Ann. Phys. (N. Y.) 9, 1 (1960).

${ }^{3} J$. R. Mika, Nucl. Soi, Eng. 11, 415 (1961).

- G. J. Mitsis, Argonne National Laboratory, ANL-6459 (1961).

S. Zelazny, J. Math. Phys. 2, 538 (1961). determining singular solutions to the integrodifferential form of the homogeneous transport equation by separation of variables in infinite space. Solutions to boundary-value problems are expanded in terms of these singular solutions. Determination of the expansion coefficients is achieved by solving singular integral equations.

The method of this paper also leads to a study of singular integral equations, which are adjoint to those derived in the applications of Case's method. For this reason our method seems to be dual to that of Case. In deriving these equations we do not use singular solutions to the integrodifferential equation form of the transport problem. Rather, we consider the familiar associated Fredholm equation which incorporates the boundary conditions for any given problem. In another paper we have shown by operational methods that this integral equation can be readily replaced by singular integral equations and linear constraints. The linear constraints come from properties of the analytic continuation of a certain function.

Our transformation to singular equations is achieved by a method which differs from the Fourier transform methods of the Weiner-Hopf analysis ${ }^{6}$ of integral operators with difference kernels on a halfline. Our method can be applied to the study of a wide class of such operators defined over an arbitrary interval. We plan to pursue this in a later paper.

We wish to emphasize one other point of our

- N. Wiener and E. Hopf, Sitzber. Preuss. Akad. Wiss. Physik. Math. Kl.1931, 696. (1931). 
analysis. Because of analytic properties of certain functions, we are able to extend the standard results of singular equation theory ${ }^{7}$ by means of analytic continuation. This leads to certain Fredholm equations which are simpler than those given by the standard theory.

We first consider in Sec. 2 the problem of anisotropic scattering in a one-dimensional slab which is of subcritical thickness $\tau$. If the slab is exposed on one face to a monodirectional beam of neutrons and a steady state is reached, the neutron source function $J$, a function of two variables which gives the distribution of neutrons in space and angle emergent from collisions, satisfies a familiar linear integral equation. ${ }^{1,8-10}$ For a subcritical reactor this integral equation can be solved by a Neumann series in an integral operator $\Lambda$. From this series it is difficult to obtain any information about the spectrum of the operator $\Lambda$.

With the assumption that the scattering function $f\left(\Omega, \Omega^{\prime}\right)$ has a finite Legendre polynomial expansion, it has been shown ${ }^{11}$ that the integral equation for $J$ can be replaced by a functional equation which relates $J$ at the symmetric depths $x$ and $\tau-x$. In Sec. 3 this equation is shown to lead to linear singular integral equations and a finite number of linear constraints.

In Sec. 4, we use singular equation theory and analytic continuation to convert the singular equations to Fredholm equations with continuous kernels. The solution to these Fredholm equations contain arbitrary constants which are determined by the linear constraints mentioned above. The determinant of this system of linear algebraic equations contains the Fredholm determinant of the operator $\Lambda$. For given $f$ and $c>1$, the critical dimension is determined by the smallest $\tau$ which makes this determinant vanish. By a limiting process we obtain the critical source function, which is an eigenfunction of the operator $\Lambda$. In fact, all eigenvalues and eigenfunctions of $\Lambda$ can be so determined. In certain cases, we also obtain solutions to the Milne problem for $\tau=\infty$. $^{1,8,10}$

In Sec. 5, specific results for the slab problem

7 N. I. Muskhelishvili, Singular Integral Equations, (P. Noordhoff Ltd., Groningen, The Netherlands, 1953).

${ }^{8} \mathrm{~K}$. M. Case, F. DeHoffmann, and G. Placzek, Introduction to the Theory of Neutron Diffusion (U. S. Government Printing Office, 1953), Vol. 1.

- S. Chandrasekhar, Radiative Transfer (Dover Publications, Inc., New York, 1960; Oxford University Press, London, 1950 ).

${ }^{10}$ E. Hopf, "Mathematical Problems of Radiative Equilibrium" (Cambridge Tracts, No. 31, 1934).

${ }_{11} \mathrm{~T}$. W. Mullikin, The RAND Corporation, RM-3558-PR, (1963) (to appear in Astrophys. J.). are obtained in the case of scattering functions linear in $\left(\Omega \cdot \Omega^{\prime}\right)$, and in the case of isotropic scattering. The results for isotropic scattering are related to previous results obtained by Mitsis ${ }^{4}$ and Zelazny, who constructed their solutions from Case's ${ }^{2}$ singular solutions to the transport equation.

In Sec. 6 it is shown that the sphere criticality problem for isotropic scattering is also easily handled by the method of this paper. In fact, a slight modification of the slab reactor results immediately gives estimates for the sphere.

\section{THE TRANSPORT EQUATION}

We shall consider a homogeneous plane-parallel reactor of finite thickness $\tau$. Depth into the slab is measured by the variable $x, 0 \leq x \leq \tau$, in a direction normal to a face of the slab and in units of a mean free path. We let $\Psi(x, \mu)$ denote the angular neutron density at depth $x$ in a direction specified by $\theta$, the inclination to the positive $x$ axis. Here $\mu=\cos \theta$.

We consider an axially symmetric beam of neutrons having unit flux incident on the slab at $x=0$ from some direction $\mu_{0} \geq 0$. The steady-state transport equation for the angular density of scattered neutrons is then ${ }^{8}$

$\mu\left[\partial \Psi\left(x, \mu, \mu_{0}\right) / \partial x\right]+\Psi\left(x, \mu, \mu_{0}\right)=J\left(x, \mu, \mu_{0}\right)$,

where the source function $J$ is given by

$$
\begin{array}{rl}
J\left(x, \mu, \mu_{0}\right)=\frac{1}{2} \int_{-1}^{1} & f(\mu, \nu) \Psi\left(x, \nu, \mu_{0}\right) d \nu \\
& +f\left(\mu, \mu_{0}\right) \exp \left(-x / \mu_{0}\right) .
\end{array}
$$

Boundary conditions result from the fact that no scattered neutrons enter the faces of the slab, and are given by

$\Psi\left(0, \mu, \mu_{0}\right)=\Psi\left(\tau,-\mu, \mu_{0}\right)=0, \quad 0 \leq \mu \leq 1$.

The last term on the right-hand side of (2.2) is the resultant source due to the reduced incident flux.

We assume that the positive scattering function $f$ in (2.2) is given by a finite expansion in Legendre polynomials,

(i) $f \geq \epsilon>0$,

(ii) $f(\mu, \nu)=c \sum_{i=0}^{N} b_{i} P_{i}(\mu) P_{i}(\nu), \quad b_{0}=1$.

For simplicity, we have assumed azimuthal symmetry. Mullikin ${ }^{11}$ has shown that the general case of azimuthal dependence leads to additional independent equations which may be handled in the same way with very little additional effort. 
From (2.1), (2.2), and (2.3) we obtain expressions for $\Psi$ in terms of $J$ by

$$
\begin{array}{r}
\Psi\left(x, \mu, \mu_{0}\right)=\frac{1}{\mu} \int_{0}^{x} \exp [(y-x) / \mu] \\
\times J\left(y, \mu, \mu_{0}\right) d y
\end{array}
$$

for $0<\mu \leq 1$, and

$$
\begin{array}{r}
\Psi\left(x, \mu, \mu_{0}\right)=-\frac{1}{\mu} \int_{x}^{\tau} \exp [(y-x) / \mu] \\
\times J\left(y, \mu, \mu_{0}\right) d y
\end{array}
$$

for $-1 \leq \mu<0$. Combined with (2.2), this gives the integral equation

$$
\begin{aligned}
& J\left(x, \mu, \mu_{0}\right)=f\left(\mu, \mu_{0}\right) \exp \left(-x / \mu_{0}\right) \\
& +\frac{1}{2} \int_{0}^{1} f(\mu, \sigma) \int_{0}^{x} \exp [(y-x) / \sigma] J\left(y, \sigma, \mu_{0}\right) d y \frac{d \sigma}{\sigma} \\
& -\frac{1}{2} \int_{-1}^{0} f(\mu, \sigma) \int_{x}^{\tau} \exp [(y-x) / \sigma] J\left(y, \sigma, \mu_{0}\right) d y \frac{d \sigma}{\sigma} .
\end{aligned}
$$

We abbreviate this equation by

$$
J=c \Lambda(J)+B .
$$

It is easily shown that because of (2.4) the operator $\Lambda$ is strictly positive. ${ }^{12}$ This implies that $\Lambda$ has a real positive eigenvalue $\bar{\lambda}$ of unit multiplicity, which dominates the modulus of all other eigenvalues. This eigenvalue is a function of the parameters $\tau, b_{1}, \cdots, b_{N}$. The eigenfunction $\rho$ associated with $\bar{\lambda}$ can be chosen to be uniformly positive. ${ }^{12}$

We will need the following result (see $\mathrm{Hopf}^{10}$ ). Let the constants $b_{1}, \cdots, b_{N}$ be restricted by (2.4). Then the maximum eigenvalue $\bar{\lambda}$ of $\Lambda$ satisfies

$$
\bar{\lambda}\left(\tau, b_{1}, \cdots, b_{N}\right)<1, \quad 0 \leq \tau<\infty .
$$

If the constant $c$ is restricted by

$$
c \bar{\lambda}<1
$$

Eq. (2.8) has the unique solution given by the Neumann series

$$
J=\sum_{n=0}^{\infty} c^{n} \Lambda^{n}(B)
$$

The solution is an analytic function of the complex variable $\mu_{0}$ for all $0<\left|\mu_{0}\right|<\infty$ with a pole of order $N$ at $\infty$, and real valued and nonnegative for real $\mu_{0}, \mu_{0} \neq 0$.

We observe from (2.4) that

$$
f(\mu,-\nu)=f(-\mu, \nu) .
$$

${ }^{12}$ S. Karlin, J. Math. Mech. 8, 907 (1959).
It then follows easily from (2.7) that

$$
J\left(\tau-x,-\mu, \mu_{0}\right)=\exp \left(-\tau / \mu_{0}\right) J\left(x, \mu,-\mu_{0}\right) .
$$

The solution given by (2.11) is computationally unsatisfactory for reactors which are near criticality because of the slow rate of convergence of the series. We shall solve (2.8) for subcritical reactors by replacing (2.8) by singular linear integral equations which give a more satisfactory procedure of numerical solution. From the solution of these equations we shall obtain a method for computing $\bar{\lambda}\left(\tau, b_{1}\right.$, $\left.\cdots, b_{N}\right)$ and hence the criticality condition

$$
c \bar{\lambda}\left(\tau, b_{1}, \cdots, b_{N}\right)=1 .
$$

If we then take the solution $J$ to the subscritical problem and let $c$ increase to criticality we shall find, by dominance of $\bar{\lambda}$,

$$
\lim _{c \bar{\lambda} \rightarrow 1}(1-c \bar{\lambda}) J=\rho .
$$

This function $\rho$ is an eigenfunction of the operator $\Lambda$ and satisfies

$$
\rho=c \Lambda(\rho) .
$$

It represents the angular density of source neutrons in space and direction in a critical reactor.

It is easily shown that $\Lambda$, as defined by (2.7), has a kernel which is square integrable in the variables $x, y, \mu$, and $\nu$ for $0 \leq x, y \leq \tau,-1 \leq \mu$, $\nu \leq 1$. Therefore the eigenvalues of $\Lambda$ are discrete, although not necessarily real, since $\Lambda$ is not Hermitian in general. From our solution to singular integral equations, we can determine all the eigenvalues and eigenfunctions of $\Lambda$.

\section{LINEAR SINGULAR EQUATIONS}

In the previous section, we arrived at an integral equation (2.7) whose solution is the source function $J$ for a scattering problem. Mullikin ${ }^{11}$ has shown that (2.7) can be replaced by another equation in which the roles of the independent variables $x$ and $\mu$ and the parameter $\mu_{0}$ are interchanged. We merely outline this derivation.

In (2.7) the variable $\mu_{0}$ is usually restricted by $0 \leq \mu_{0} \leq 1$ as the cosine of an angle in the interval $\left[0, \frac{1}{2} \pi\right]$. In the term $f\left(\mu, \mu_{0}\right)$ exp $\left(-x / \mu_{0}\right)$ let us replace $\mu_{0}$ in the exponential by a complex number $z, z \neq 0$. We then consider the equation

$$
\begin{aligned}
K\left(x, \mu, \mu_{0}, z\right)=f\left(\mu, \mu_{0}\right) & \exp (-x / z) \\
& +c \Lambda(K)\left(x, \mu, \mu_{0}, z\right) .
\end{aligned}
$$

It is easily shown that this equation has a solution which is analytic in $z$ for $|z|>0$. For $z=\mu_{0}$ this solution agrees with the solution $J$ to (2.7). 
We now apply the integral operator $\Lambda$ to both sides of (3.1). This can readily be seen to give an equation for $\Lambda(K)$ which is similar to (3.1) except that the forcing term consists of integrals over the parameters $\mu_{0}$ and $z$. Applying $(I-c \Lambda)^{-1}$ to this equation, we obtain a representation of $\Lambda(K)$ in terms of $K$ and $J$. When this is combined with (3.1) we obtain

$$
\begin{aligned}
& K(x, \mu, \nu, z) \\
& -\frac{c z}{2} \int_{-1}^{1} \frac{K(x, \mu, \sigma, z)-K(x, \mu, z, z)}{z-\sigma} f(\nu, \sigma) d \sigma \\
& =f(\mu, \nu) \exp (-x / z) \\
& +\frac{c z}{2} \int_{0}^{1} \frac{J(x, \mu, z)-J(x, \mu, \sigma)}{z-\tau} f(\nu, \sigma) d \sigma \\
& +\frac{c z}{2} \int_{-1}^{0} \frac{J(x, \mu, z)-\exp (-\tau / z) J(\tau-x,-\mu,-\sigma)}{z-\sigma} \\
& \times f(\nu, \sigma) d \sigma .
\end{aligned}
$$

If we now consider fixed values of the variables $x, \mu$, and $z$, then the left-hand side of (3.2) contains a linear operator $L_{z}$ which acts only on the third argument of the function $K$. The variable $\nu$ enters the right-hand side of (3.2) only in the function $f(\nu, \sigma)$. If we assume that $f$ has a finite expansion in Legendre polynomials, then it can be shown ${ }^{11}$ that the operator $\left(I-L_{z}\right)$ has an inverse and that (3.2) can be solved for $K$ in terms of $J$.

After solving (3.2) for $K$, we again set $\nu$ equal to $z$ to obtain

$$
\begin{aligned}
& J(x, \mu, z)=k(\mu, z) \exp (-x / z) \\
& -\frac{z}{2} \int_{0}^{1} \frac{J(x, \mu, z)-J(x, \mu, \sigma)}{z-\sigma} k(\sigma, z) d \sigma \\
& +\frac{z}{2} \int_{-1}^{0} \frac{J(x, \mu, z)-\exp (-\tau / z) J(\tau-x,-\mu,-\sigma)}{z-\sigma} \\
& \times k(\sigma, z) d \sigma .
\end{aligned}
$$

Notice that now $x$ and $\mu$ are merely parameters. The function $k$ is given by

$$
k(\mu, z) \equiv c \sum b_{i} P_{i}(\mu) h_{i}(z),
$$

where $h_{i}$ are defined recursively by

$$
h_{0}(z)=1, \quad h_{1}(z)=(1-c) z,
$$

$$
(j+1) h_{i+1}(z)+j h_{i-1}(z)
$$

$$
=z\left[(2 j+1)-c b_{i}\right] h_{i}(z) .
$$

This formula for the $h_{i}$ is obtained by $\mathrm{Mika}^{3}$ by a different method than that used by Mullikin. ${ }^{11}$

We are primarily interested in $J(x, \mu, z)$ for $z$ in the interval $[0,1]$. Once this is determined, we have the analytic continuation of $J$ to complex $z$ given by (3.3). For the moment we restrict $z$ to complex values outside the interval $[-1,1]$ and rearrange (3.3) as

$$
\begin{aligned}
\lambda(z) J(x, \mu, z) & =k(\mu, z) \exp (-x / z) \\
& -\frac{z}{2} \int_{0}^{1} \frac{k(\sigma, z) J(x, \mu, \sigma)}{z-\sigma} d \sigma \\
& -\frac{z}{2} \exp (-\tau / z) \int_{-1}^{0} \frac{k(\sigma, z) J(\tau-x,-\mu,-\sigma)}{z-\sigma} d \sigma .
\end{aligned}
$$

The function $\lambda$ is defined by

$\lambda(z)=1+z \int_{-1}^{1} \frac{\gamma(\sigma) d \sigma}{\sigma-z}, \quad z \notin[-1,1]$,

where

$$
\gamma(\sigma)=\frac{1}{2} k(\sigma, \sigma) .
$$

To obtain (3.7) from (3.4) we have used the result given by Mullikin ${ }^{11}$ that

$$
\int_{-1}^{1} \frac{k(\sigma, z)-k(\sigma, \sigma)}{\sigma-z} d \sigma=0 .
$$

It is also shown by Mullikin ${ }^{11}$ that

$$
k(\mu, z)=k(-\mu,-z)
$$

and hence that

$$
\gamma(\sigma)=\gamma(-\sigma) .
$$

For special functions $f$, the function $\gamma$ has been called the "characteristic function" by Chandrasekhar."

The expression (3.4) for $k$ shows that Eq. (3.6) can be expanded in terms of Legendre polynomials in $\mu$ to give $(N+1)$ independent equations. Since this expansion can be done at any stage of our treatment, we simply study the single equation (3.6) with $\mu$ as a parameter.

The first piece of information to be gained from the functional equation (3.6) is a set of linear constraints. We want a solution to (3.6) which is analytic in $z$ for $0<|z|<\infty$. If the function $\lambda$ vanishes at some point, or set of points, $z_{i}$ with $z_{i}$ finite and outside the interval $[-1,1]$, then for $J$ to be analytic the right-hand side of (3.6) must vanish to the same order as $\lambda$ at these points. This gives linear constraints such as

$$
\begin{aligned}
& k\left(\mu, z_{i}\right) \exp \left(-x / z_{i}\right) \\
& =\frac{z_{i}}{2} \int_{0}^{1} \frac{k\left(\sigma, z_{i}\right) J(x, \mu, \sigma)}{z_{i}-\sigma} d \sigma \\
& \quad+\exp \left(-\tau / z_{i}\right) \frac{z_{i}}{2} \int_{-1}^{0} \frac{k\left(\sigma, z_{i}\right) J(\tau-x,-\mu,-\sigma)}{z_{i}-\sigma} d \sigma,
\end{aligned}
$$


for $z_{i}$ satisfying

$$
\lambda\left(z_{i}\right)=0, \quad \lambda^{\prime}\left(z_{i}\right) \neq 0 .
$$

Complications arise if $\lambda$ vanishes at infinity. It follows easily from (3.11), by induction on $n$, that for $n \geq 1$

$$
\begin{aligned}
h_{n}(z)=P_{n}(z) \prod_{i=0}^{n-1}(1- & \left.\frac{c b_{i}}{2 i+1}\right) \\
& + \text { lower order } P_{i} .
\end{aligned}
$$

Then from (3.7), (3.4), and (3.14), we have

$$
\begin{aligned}
\lambda(\infty) & =(1-c) \\
& -c \sum_{i=1}^{N} \frac{b_{i}}{2 i+1} \prod_{i=0}^{i-1}\left(1-\frac{c b_{i}}{2 j+1}\right) .
\end{aligned}
$$

Again, by induction on $N$, it readily follows that

$$
\lambda(\infty)=\prod_{i=0}^{N}\left(1-\frac{c b_{i}}{2 i+1}\right) .
$$

Now formula (3.6) gives the analytic continuation of $J(x, \mu, z)$ off the interval $-1 \leq z \leq 1$, and $J$ must be so chosen on the interval $[-1,1]$ that this function is analytic in $0<|z|<\infty$ with a pole of order $N$ at infinity. From (3.14) and (3.16) we see that if $\lambda(\infty) \neq 0$, then $h_{N}(z)$ is of degree $N$, and hence also $k(\mu, z)$ and $J$ are of degree $N$ in $z$, giving the correct-order pole at infinity. However, if $c b_{N}=2 N+1$, then $k$ is of degree $N$ but $\lambda(\infty)=0$, and conditions have to be imposed to prevent $J$ having a higher-order pole than $N$ at infinity. Also, if some $c b_{i}=2 i+1, i<N$, it follows easily that $k(\mu, z)$ is at most of degree $(N-1)$ in $z$ and that $\lambda$ vanishes at infinity at least to order two. Then conditions again have to be imposed to give $J$ the correct behavior at infinity.

It should be remembered that the constants $b_{i}$ are not completely arbitrary but are restricted by the condition (2.4) of positivity of the scattering function $f$. However, it seems difficult to relate these restrictions in general to possibilities discussed above.

Further calculations will require the frequent use of Plemelj's formulas. ${ }^{11}$ The function $\varphi$, defined by

$$
\varphi(z)=\frac{1}{2 \pi i} \int_{a}^{b} \frac{f(\sigma) d \sigma}{\sigma-z},
$$

is analytic in the complex plane cut along $[a, b]$. Provided $f$ satisfies a Hölder condition on $(a, b)$, Plemelj's formulas give the limits $\varphi^{+}$and $\varphi^{-}$of $\varphi$ from the upper and lower half-planes, respectively, as $z$ tends to $\nu, a<\nu<b$ :

$$
\begin{gathered}
\varphi^{+}(\nu)=\frac{1}{2} f(\nu)+\frac{1}{2 \pi i} \int_{a}^{b} \frac{f(\sigma) d \sigma}{\sigma-\nu}, \\
\varphi^{-}(\nu)=-\frac{1}{2} f(\nu)+\frac{1}{2 \pi i} \int_{a}^{b} \frac{f(\sigma) d \sigma}{\sigma-\nu} .
\end{gathered}
$$

The integrals are computed as Cauchy principal values.

Remembering that $J$ is to be analytic in $0<$ $|z|<\infty$, we now apply Plemelj's formulas to compute the limits in (3.6) as $z$ tends to $\nu$ with $-1<\nu<1$, $\nu \neq 0$. Adding the limits from the lower and upper half-planes, we obtain a singular integral equation for $J(x, \mu, \nu)$ on the interval $-1<\nu<1$ :

$$
\begin{aligned}
\lambda_{0}(\nu) J(x, \mu, \nu) & =k(\mu, \nu) \exp (-x / \nu) \\
& -\frac{\nu}{2} \int_{0}^{1} \frac{k(\sigma, \nu) J(x, \mu, \sigma) d \sigma}{\nu-\sigma} \\
-\exp (-\tau / \nu) & \frac{\nu}{2} \int_{-1}^{0} \frac{k(\sigma, \nu)}{\nu-\sigma} J(\tau-x,-\mu,-\sigma) d \sigma .
\end{aligned}
$$

The singular integrals are computed as Cauchy principal values, and $\lambda_{0}$ is given by

$$
\lambda_{0}(\nu)=1+\nu \int_{-1}^{1} \frac{\gamma(\sigma)-\gamma(\nu)}{\sigma-\nu} d \sigma-\nu \gamma(\nu) \ln \frac{1+\nu}{1-\nu}
$$

To simplify the subsequent analysis, we choose to write (3.19) as two uncoupled equations on the interval $(0,1)$. We do this by defining two functions by

$$
\begin{gathered}
P(x, \mu, \nu)=J(x, \mu, \nu)+J(\tau-x,-\mu, \nu), \\
Q(x, \mu, \nu)=J(x, \mu, \nu)-J(\tau-x,-\mu, \nu), \\
0<\nu<1 .
\end{gathered}
$$

The equations for $P$ and $Q$ are then

$$
\begin{aligned}
& \lambda_{0} P=l_{1}+V(P)-U(P), \\
& \lambda_{0} Q=l_{2}+V(Q)+U(Q) .
\end{aligned}
$$

For brevity, we have defined two integral operators, $V$ and $U$, by

$$
\begin{array}{r}
V(J)(x, \mu, \nu)=\frac{\nu}{2} \int_{0}^{1} \frac{k(\sigma, \nu) J(x, \mu, \sigma)}{\sigma-\nu} d \sigma \\
U(J)(x, \mu, \nu)=\exp (-\tau / \nu) \frac{\nu}{2} \int_{0}^{1} \frac{k(-\sigma, \nu)}{\sigma+\nu} \\
\times J(x, \mu, \sigma) d \sigma
\end{array}
$$

for $0<y<1$, and we have introduced the known functions $l_{1}$ and $l_{2}$ given by 


$$
\begin{aligned}
& l_{1}(x, \mu, \nu) \equiv k(\mu, \nu) \exp (-x / \nu) \\
&+k(\mu,-\nu) \exp [(x-\tau) / \nu] \\
& l_{2}(x, \mu, \nu) \equiv k(\mu, \nu) \exp (-x / \nu) \\
&-k(\mu,-\nu) \exp [(x-\tau) / \nu] .
\end{aligned}
$$

In the next section we use techniques of analytic function theory to convert (3.22) to Fredholm equations with continuous kernels. Although Mika, ${ }^{3}$ by using Muskhelishvili's standard method, ${ }^{7}$ has already treated singular equations of this type for a semi-infinite medium, which are adjoint in the singular part of (3.22), we choose to employ a slight modification of the standard method. In this way, we take advantage of certain simplifications resulting from the analytic properties of the various functions.

\section{FREDHOLM EQUATIONS}

In this section, then, we consider the singular integral equation

$$
\lambda_{0}(\nu) R(\nu)=F(\nu)+\frac{\nu}{2} \int_{0}^{1} \frac{k(\sigma, \nu) R(\sigma) d \sigma}{\sigma-\nu},
$$

which is Eq. (3.22) for $P$ (or $Q$ ), with $l_{1}$ (or $l_{2}$ ) and the bounded operator $U$ included in the definition of $F$. That is, if (4.1) is to represent (3.22) for $P$, then

$$
F(\nu)=l_{1}(x, \mu, \nu)-U(P)(x, \mu, \nu),
$$

or, if it is to represent (3.22) for $Q$, we have

$$
F(\nu)=l_{2}(x, \mu, \nu)+U(Q)(x, \mu, \nu) .
$$

Since $x$ and $\mu$ are merely parameters in our equations, we will not display them. We will treat $F$ as a known function and invert the singular operator by a slightly modified form of the theory of singular integral equations. ${ }^{7}$ Finally, we will obtain a regular Fredholm equation for $F$.

Since $k$ is a polynomial, a sectionally holomorphic function $\varphi(z)$ is defined by

$$
\varphi(z)=\frac{1}{2 \pi i} \int_{0}^{1} \frac{k(\sigma, z) R(\sigma) d \sigma}{\sigma-z} .
$$

By Plemelj's formulas it is easily verified that we can write (4.1) as

$\lambda^{-}(\nu) \varphi^{+}(\nu)=\lambda^{+}(\nu) \varphi^{-}(\nu)+2 \gamma(\nu) F(\nu), 0 \leq \nu \leq 1$,

where

$$
\begin{aligned}
& \lambda^{+}(\nu)=\lambda_{0}(\nu)+\pi i \nu \gamma(\nu), \\
& \lambda^{-}(\nu)=\lambda_{0}(\nu)-\pi i \nu \gamma(\nu) .
\end{aligned}
$$

We divide (4.5) by $\lambda^{-}$and rewrite it as $\varphi^{+}(v)-\left[\lambda^{+}(\nu) / \lambda^{-}(v)\right] \varphi^{-}(\nu)=2 \gamma(v) F(v) / \lambda^{-}(\nu)$.

By standard methods $_{2}^{7}$ we now construct a nonvanishing sectionally holomorphic function $E(z)(z \in[0,1])$, which solves the homogeneous equation

$$
E^{+}(\nu) / E^{-}(\nu)=\lambda^{+}(\nu) / \lambda^{-}(\nu) .
$$

We find that

$$
E(z)=\exp [\Gamma(z)] /(1-z)^{\alpha}
$$

is the required solution, where the function $\Gamma$ is defined by

$$
\Gamma(z)=\int_{0}^{1} \frac{\theta(t) d t}{t-z}
$$

and

$$
\theta(t)=\frac{1}{\pi} \tan ^{-1} \frac{\pi t \gamma(t)}{\lambda_{0}(t)}, \quad 0 \leq t \leq 1 .
$$

It can be shown ${ }^{3,11}$ that the index, $\alpha$, is a nonnegative integer given by

$$
\alpha=\theta(1) \leq N+1 \text {. }
$$

We will henceforth assume that

$$
\lambda_{0}^{2}(\nu)+[\nu \gamma(\nu)]^{2} \neq 0, \quad 0 \leq \nu \leq 1 .
$$

If (4.13) does not hold, the index $\alpha$ is increased and is accompanied by an equal increase in the number of constraints on the equation for $J$ [see (3.12)], where the additional $z_{i}$ are just the zeros of the left-hand side of (4.13).

Inserting (4.8) into (4.7) gives

$$
\varphi^{+}(\nu)-\left[E^{+}(\nu) / E^{-}(\nu)\right] \varphi^{-}(\nu)=2 \gamma(\nu) F(\nu) / \lambda^{-}(\nu),
$$

or

$$
\frac{\varphi^{+}(\nu)}{E^{+}(\nu)}-\frac{\varphi^{-}(\nu)}{E^{-}(\nu)}=\frac{2 \gamma(\nu) F(\nu)}{\lambda^{-}(\nu) E^{+}(\nu)}
$$

Now consider the function $G$

$G(z)=\frac{\varphi(z)}{E(z)}-\frac{1}{2 \pi i} \int_{0}^{1} \frac{2 \gamma(\sigma) F(\sigma) d \sigma}{\lambda^{-}(\sigma) E^{+}(\sigma)(\sigma-z)}$

$G$ is clearly analytic in the finite cut plane and, from (4.15), we find that $G$ is continuous across the cut and hence is analytic in the entire finite plane. Finally, if

$$
\beta \equiv \text { degree of } z \quad \text { in } k(\sigma, z),
$$

then, by considering the behavior of the right-hand side of (4.16) at infinity, we conclude that $G$ is a polynomial of degree $\alpha+\beta-1$ at most, which 
we choose to write as

$$
G(z)=\frac{p_{\alpha+\beta-1}(z)}{\pi i}=\frac{1}{\pi i} \sum_{i=0}^{\alpha+\beta-1} a_{i} z^{i}
$$

Therefore, (4.16) becomes after multiplication by $E(z)$

$$
\begin{array}{r}
\varphi(z)=\frac{E(z)}{\pi i} \int_{0}^{1} \frac{\gamma(\sigma) F(\sigma) d \sigma}{\lambda^{-}(\sigma) E^{+}(\sigma)(\sigma-z)}+\frac{1}{\pi i} \\
\times E(z) p_{\alpha+\beta-1}(z) .
\end{array}
$$

The standard singular integral equation theory concludes at this point by equating $\varphi^{+}-\varphi^{-}$as computed from (4.4) and from (4.19). If this is done for $R$ set equal to $P$ (or $Q$ ) and for $F$ replaced by its definition in terms of $P$ (or $Q$ ), then we obtain a Fredholm equation for $P$ (or $Q$ ) on the interval $[0,1]$. The kernel is such an equation is complicated. Fortunately we can use the analytic properties of the functions $P$ and $Q$ and the polynomial nature of the kernel $k$ to obtain simpler Fredholm equations for the function $F$ given by (4.2) [or by (4.3)]. This will also lead to an evaluation of the coefficients $a_{i}$ in the polynomial $p_{\alpha+\beta-1}$.

We, therefore, specialize to the case where $R=P$, and $F$ is defined by (4.2). By an obvious modification we can apply our results to the function $Q$.

Using the definition (3.23) of $U$ and $V$, we see that $F(z)$, defined by (4.2), is simply related to $\varphi(-z)$ in (4.4). But $\varphi(-z)$ can also be computed by (4.19), and this gives us the following equation for $F$ :

$$
\begin{aligned}
& F(z)=l_{1}(z)-z e^{-\tau / z} E(-z) \\
& \quad \times\left[\int_{0}^{1} \frac{\gamma(\sigma) F(\sigma) d \sigma}{\lambda^{-}(\sigma) E^{+}(\sigma)(\sigma+z)}+\sum_{i=0}^{\alpha+\beta-1} a_{i}(-z)^{i}\right] .
\end{aligned}
$$

We define the linear operator $L$ by

$$
L(F)(z)=z e^{-\tau / z} E(-z) \int_{0}^{1} \frac{\gamma(\sigma) F(\sigma) d \sigma}{\lambda^{-}(\sigma) E^{+}(\sigma)(\sigma+z)},
$$

and choose to write $(4.20)$ as the following set of integral equations:

$$
\begin{gathered}
\tilde{g}(z)=l_{1}(z)-L(\tilde{g})(z), \\
g_{i}(z)=(-z)^{i+1} e^{-\tau / z} E(-z)-L\left(g_{i}\right)(z), \\
i=0, \cdots, \alpha+\beta-1 .
\end{gathered}
$$

Because of the linearity of $L$, we clearly have

$$
F(z)=\tilde{g}(z)+\sum_{i=0}^{\alpha+\beta-1} a_{i} g_{i}(z) .
$$

For $z$ in the interval $[0,1]$, the Fredholm operator $L$ has a continuous kernel. If the norm of $L$ satisfies
$\|L\|<1$ in some Banach space, then Eqs. (4.22) may be solved by iteration. These iterations will converge uniformly if

$\max _{0 \leq \nu \leq 1}\left[\nu e^{-\tau / \nu} E(-\nu) \int_{0}^{1} \frac{|\gamma(\sigma)| d \sigma}{E^{+}(\sigma) \lambda^{-}(\sigma)(\sigma+\nu)}\right]<1$.

For any given $\gamma$ this inequality will certainly hold for all sufficiently large $\tau$. We will show below that for certain $\gamma$ this holds for all $\tau \geq 0$. The convergence of the iteration scheme for all $\gamma$ and all $\tau \geq 0$ is an open question.

If we let $\mathcal{C}$ denote a circle centered at the origin and enclosing the cut along $[0,1]$ and the point $z$, we can show ${ }^{13}$ by contour integration that

$$
\begin{aligned}
\int_{0}^{1} & \frac{\gamma(\sigma) d \sigma}{E^{+}(\sigma) \lambda^{-}(\sigma)(\sigma-z)} \\
= & \frac{1}{z E(0)}-\frac{1}{z E(z)}-\frac{1}{2 \pi i} \int_{\mathfrak{e}} \frac{d w}{E(w) w(w-z)} \\
= & \frac{1}{z E(0)}-\frac{(1-z)^{\alpha} \exp [-\Gamma(z)]}{z} \\
& \quad-\sum_{i=0}^{\alpha-1} \frac{z^{i}}{2 \pi i} \int_{\mathfrak{e}} \frac{\exp [-\Gamma(w)](1-w)^{\alpha} d w}{w^{j+2}}
\end{aligned}
$$

The series in $z$ terminates since coefficients of higherorder terms can be shown to vanish by choosing e with arbitrarily large radius.

Now the left-hand side of (4.25) vanishes at infinity, and so must the right-hand side. The principle part of the Laurent expansion about infinity of $\exp [-\Gamma(z)](1-z)^{\alpha} / z$ can be used to evaluate the various integrals around $\mathfrak{e}$ in terms of such quantities as $\int_{0}^{1} t^{n} \theta(t) d t$. We shall abbreviate the polynomial part of $(4.25)$ by $q(z)_{\alpha-1}$, a polynomial of degree $\alpha-1$. Then we have

$$
\begin{array}{r}
\nu E(-\nu) \int_{0}^{1} \frac{\gamma(\sigma) d \sigma}{E^{+}(\sigma) \lambda^{-}(\sigma)(\sigma+\nu)}=1-\frac{E(-\nu)}{E(0)} \\
-\nu E(-\nu) q(-\nu)_{\alpha-1} .
\end{array}
$$

As a first application of this identity, we suppose that $\gamma$ is positive in the interval $[0,1]$. It follows readily $^{13}$ that $\alpha=1$ and that, by (4.26) and the facts that $E(-\nu)$ and $E^{+}(\nu) \lambda^{-}(\nu)$ are positive for all $\gamma$,

$$
\begin{array}{r}
0 \leq \nu E(-\nu) \int_{0}^{1} \frac{\gamma(\sigma) d \sigma}{E^{+}(\sigma) \lambda^{-}(\sigma)(\sigma+\nu)}<1, \\
0 \leq \nu \leq 1 .
\end{array}
$$

${ }_{13}$ T. W. Mullikin, The RAND Corporation, RM-3376JPL (1962) (to appear in Trans. Am. Math. Soc.). 
In this case of positive $\gamma$ on $[0,1]$, condition (4.24) is valid, the operator $L$ on $[0,1]$ is contracting for all $\tau \geq 0$, and Eqs. (4.22) can be solved by iteration for all $\tau \geq 0$.

For the remainder of this paper we assume that the Fredholm equations (4.22) have been solved for the functions $\tilde{g}$ and $g_{i}$ on the interval $[0,1]$. Once these functions are computed on $[0,1]$, their analytic continuation to complex $z$ outside the cut $[-1,0]$ is given again by (4.22).

We now turn our attention to the evaluation of the $\alpha+\beta$ constants $a_{i}$, which is needed to determine the function $F$.

In Sec. 3 we noted that linear constraints are obtained by demanding regularity of $J$ at the zeros of $\lambda$ in the plane cut along $[-1,1]$. By an application of the argument principle to $\lambda$, it can be shown ${ }^{3,11}$ that the number of zeros of $\lambda$ equals $2 \alpha$. Also by (3.5) and the fact that $\gamma$ is an even function, we see that the zeros of $\lambda$ occur in symmetric pairs $\pm z_{i}$. Now from (2.13) and (3.21), we see that we seek solutions $P$ and $Q$ which satisfy

$$
\begin{gathered}
P(x, \mu, \nu)=\exp (-\tau / \nu) P(x, \mu,-\nu), \\
Q(x, \mu, \nu)=-\exp (-\tau / \nu) Q(x, \mu,-\nu) .
\end{gathered}
$$

Therefore, independent conditions are obtained by requiring regularity of $P$ and $Q$ at only one of each of the pairs of roots $\pm z_{i}$, and this is sufficient to obtain regularity of $J(x, \mu, z)$ at all the zeros $\pm z_{i}$.

We assume, for simplicity, that $\lambda$ has only simple zeros which are finite and outside the cut $[-1,1]$, i.e., we assume in particular that the constant $c$ is such that $\lambda(\infty) \neq 0$ as given by (3.16). Then from (3.12), (3.21), (3.23), and (4.2), we see that for the function $F$ we obtain $\alpha$ linear constraints

$F\left(z_{i}\right)+\exp \left(-\tau / z_{i}\right)\left[F\left(-z_{i}\right)-l_{1}\left(-z_{i}\right)\right]=0$

for $i=1, \cdots, \alpha$. Using (4.22) and (4.23), we may write this as

$$
\begin{aligned}
& \sum_{i=0}^{\alpha+\beta-1} D_{i j}\left(\gamma, c, b_{1}, \cdots, b_{N}\right) a_{i}=l_{1}\left(z_{i}\right) \\
& -L(\tilde{g})\left(z_{i}\right)-e^{-\tau / z i} L(\tilde{g})\left(-z_{i}\right), \quad i=1, \cdots, \alpha,
\end{aligned}
$$

where

$$
\begin{aligned}
D_{i i}(\tau, c, & \left.b_{1}, \cdots, b_{N}\right) \\
= & z_{i}^{i+1} E\left(z_{i}\right)+\left(-z_{i}\right)^{i+1} e^{-\tau / z} t E\left(-z_{i}\right) \\
& -L\left(g_{i}\right)\left(z_{i}\right)-e^{-\tau / z i} L\left(g_{i}\right)\left(-z_{i}\right) .
\end{aligned}
$$

We also require $\beta$ additional equations involving the $a_{i}$ to completely determine $F$. These equations appear to be obtained most easily by investigating
(4.19) at the zeros of $\gamma$. Assume that there are $\eta$ pair of zeros, $\pm \zeta_{i}$, i.e.,

$$
\gamma\left( \pm \zeta_{i}\right)=0 \quad i=1, \cdots, \eta .
$$

Using (4.4) for $\varphi$, we multiply (4.19) by $\pi i$ and write it for $\nu= \pm \zeta_{i}$,

$$
\begin{aligned}
& \frac{1}{2} \int_{0}^{1} \frac{k\left(\sigma, \pm \zeta_{i}\right) P(\sigma) d \sigma}{\sigma \mp \zeta_{i}} \\
& \quad=E\left( \pm \zeta_{i}\right) \int_{0}^{1} \frac{\gamma(\sigma) f(\sigma) d \sigma}{\lambda^{-}(\sigma) E^{+}(\sigma)\left(\sigma \mp \zeta_{i}\right)} \\
& \quad+E\left( \pm \zeta_{i}\right) p_{\alpha+\beta-1}\left( \pm \zeta_{i}\right), \quad i=1, \cdots, \eta
\end{aligned}
$$

Since $\gamma(\sigma)=\frac{1}{2} k(\sigma, \sigma)$, we see that $k\left(\sigma, \zeta_{i}\right)$ is a polynomial in $\sigma$ which vanishes at $\zeta_{i}$. We may then write

$$
\frac{k\left(\sigma, \pm \zeta_{i}\right)}{\sigma \mp \zeta_{i}}=\sum_{i=0}^{\delta-1} w_{i i}\left(\zeta_{i}\right)( \pm 1)^{i+1} \sigma^{i}
$$

where the $w_{i j}$ are polynomials and $\delta$ is the degree of $\sigma$ in $k(\sigma, \nu)$. Denoting the moments of $P$ by

$$
m_{i}(P)=\int_{0}^{1} \sigma^{i} P(\sigma) d \sigma,
$$

and using the definition (4.21) of $L$, we find that (4.33) becomes

$$
\begin{gathered}
\frac{1}{2} \sum_{i=0}^{\delta-1} w_{i j}\left(\zeta_{i}\right)( \pm 1)^{i+1} m_{j}(P)=\mp \frac{e^{-\tau / 5 i}}{\zeta_{i}} L(F)\left(-\zeta_{i}\right) \\
+E\left( \pm \zeta_{i}\right) p_{\alpha+\beta-1}\left( \pm \zeta_{i}\right)
\end{gathered}
$$

Equations (4.36) are $2 \eta$ in number. From the results (3.4) and (3.5) we can show that, except in special cases,

$$
\delta+\beta=2 \eta .
$$

For a given value of $c$, there is at most one value $b_{N-1}$ such that $2 \eta<\delta+\beta$. In such a case, one must obtain additional equations for the constants. This is done, for example, by expanding (4.19) in a Laurent series about infinity and equating the coefficients of $z^{n}$. Or one may approach the special value of $b_{N-1}$ as a limit of a sequence of cases where (4.37) holds.

In general, therefore, the $\delta$ quantities $m_{j}(P)$ may be eliminated from the $\delta+\beta$ equations (4.36), leaving $\beta$ algebraic equations for the $a_{i}$. These equations combined with the $\alpha$ equations (4.30) will uniquely determine (at least for the subcritical reactor) the constants $a_{i}(i=0, \cdots, \alpha+\beta-1)$. We write these equations in matrix notation,

$$
\mathbf{M}\left(\gamma, c, b_{1}, \cdots, b_{N}\right) \mathbf{a}=\mathbf{v}(x, \mu, \gamma) .
$$


(We suppress the dependence of the vector $\nabla$ upon $\left.c, b_{1}, \cdots, b_{N}\right)$.

To gain further insight into the problem, we choose to solve (4.38) by expanding $\nabla$ in terms of the eigenvectors of $\mathbf{M}$. For simplicity, we will assume that $\mathbf{M}$ has $\alpha+\beta$ distinct eigenvalues so that this expansion is possible. However, the following results for the critical thickness and flux hold even if $\mathbf{M}$ has eigenvalues with multiplicity greater than one. We therefore proceed by writing

$$
\mathbf{v}=\sum_{i=0}^{\alpha+\beta-1} \tilde{v}_{i}(x, \mu, \gamma) \xi_{i}\left(\tau, c, b_{1}, \cdots, b_{N}\right)
$$

where the $\xi_{i}$ are the normalized eigenvectors of $\mathbf{M}$ which solve the following eigenvalue problem:

$$
\mathbf{M} \xi_{i}=\epsilon_{i} \xi_{i} .
$$

We choose the eigenvalues $\epsilon_{i}$ to be so arranged that

$$
\left|\epsilon_{i+1}\right| \geq\left|\epsilon_{i}\right| \text {. }
$$

The $\tilde{v}_{i}$ are then given by

$$
v_{i}=\left(\nabla, \xi_{i}^{\dagger}\right) .
$$

The " $f$ " denotes eigenvectors of the Hermitian conjugate matrix which are defined as follows:

$$
\mathbf{M}^{\dagger} \xi_{i}^{\dagger}=\epsilon_{i}^{\dagger} \xi_{i}^{\dagger} \text {. }
$$

Using the expansion (4.39), we write the solution to $(4.37)$ as

$a=\sum_{i=0}^{\alpha+\beta-1} \frac{\tilde{v}_{i}(x, \mu, \gamma)}{\epsilon_{i}\left(\tau_{i}, c, b_{1}, \cdots, b_{N}\right)} \xi_{i}\left(\tau, c, b_{1}, \cdots, b_{N}\right)$.

This is well defined since we have been restricting our attention to singular equations among whose solutions is the bounded analytic function $J$ which satisfies (2.8) for scattering in a subcritical slab. This combined with (4.22) and (4.23) completes the determination of $F$.

From (3.6) and (3.21) we can easily show that a representation of $P$ which is analytic in $0<|z|<\infty$ is given by

$$
P(z)=[F(z)+\pi i z \phi(z)] / \lambda(z),
$$

with $\phi$ as defined by (4.4), and $R=P$. We have suppressed the parameters $x$ and $\mu$. The numerator has been so chosen as to vanish at the zeros of $\lambda$, and Plemelj's formulas readily show (4.45) to be analytic across the cut $[-1,1]$. By using (4.19) as the computation of $\phi$ and the identity (4.26), we can express $P$ in terms of $F$, for $z$ outside $[-1,0]$, by

$$
\begin{aligned}
P(z)=\frac{E(z)}{\lambda(z)} & \left\{F(z)\left(z q_{\alpha-1}(z)-1 / E(0)\right)+z p_{\alpha+\beta-1}(z)\right. \\
& \left.+z \int_{0}^{1} \frac{\gamma(\sigma)[F(\sigma)-F(z)]}{\lambda^{-}(\sigma) E^{+}(\sigma)(\sigma-z)} d \sigma\right\}
\end{aligned}
$$

A similar expression can be obtained for the $Q$ function with a different $F$ function and a different polynomial $p_{\alpha+\beta-1}$. This has reduced the scattering problem to the computation of $F$ by the solving of the Fredholm equations (4.22) and the linear algebraic equations (4.38).

As is standard in the Wiener-Hopf analysis ${ }^{8}$ we observe that, by (4.8), $\lambda(z) / E(z) E(-z)$ is bounded and analytic in the finite plane with polynomial growth at infinity, and hence a polynomial. If $\lambda(\infty) \neq 0$, we equate leading terms and zeros to write

$$
\frac{\lambda(z)}{E(z) E(-z)}=\lambda(\infty) \prod_{i=1}^{\alpha}\left(z_{i}^{2}-z^{2}\right) .
$$

By evaluating this at $z=0$, we find an expression which is valid even when $\lambda(\infty)=0$, namely

$$
\frac{E(z)}{\lambda(z)}=\frac{\exp \left[\int_{0}^{1} \frac{(t+2 z) \theta(t)}{t(t+z)} d t\right]}{\prod_{i=1}^{\alpha}\left(1-z / z_{i}\right)^{2}} .
$$

For computational purposes this is to be used in (4.46).

We wish now to extract criticality conditions from our solution for a subcritical slab. Since $J$, and hence $P$, must become infinite as criticality is reached, we see from (4.46) that this can only happen when $F$ becomes infinite. For a given set of the constants $c, b_{1}, \cdots, b_{N}$ this could conceivably happen in either of two ways. It may happen that the Fredholm equations (4.22) have solutions which become infinite as $\tau$ approaches certain critical values-we have only shown this is impossible if $\gamma(\nu)>0$ for $0 \leq \nu \leq 1$. This clearly will not be true if $c-1$ is positive and sufficiently small, for then the critical thickness will be large, and (4.24) will be satisfied for $\tau$ around the critical value.

We will restrict our attention to those cases where the Fredholm equations (4.22) have bounded solutions for all $\tau$ of interest. Then $F$, as given by (4.23), can become infinite only by having the coefficients $a_{i}$ become infinite. By (4.44), this means that the eigenvalue $\varepsilon_{0}$ of $\mathbf{M}$ of minimum modulus approaches zero as criticality is attained. To avoid the eigenvalue analysis of $\mathbf{M}$, we can define the 
critical dimension $\tau_{c}$ as that minimum $\tau$ such that the determinant of $\mathbf{M}$ vanishes,

$$
\operatorname{det} \mathbf{M}\left(\tau, c, b_{1}, \cdots, b_{N}\right)=\mathbf{0} .
$$

From (4.23), (4.44), and (4.46), we also obtain the result that

$$
\begin{aligned}
& \lim _{\tau \rightarrow \tau_{c}} \epsilon_{0}\left(\tau, c, b_{1}, \cdots, b_{N}\right) P(x, \mu, z) \\
& =C\left(z, \tau_{c}, b_{1}, \cdots, b_{N}\right) \tilde{v}_{0}\left(x, \mu, \tau_{c}\right),
\end{aligned}
$$

where $C$ is a function of $z$ which is not identically zero. From (2.7) we see that

$$
\begin{gathered}
P(x, \mu, z)=c \Lambda(P)(x, \mu, z)+f(\mu, z) \exp (-x / z) \\
+f(-\mu, z) \exp [(x-\tau) / z] .
\end{gathered}
$$

We multiply this by $\epsilon_{0}\left(\tau, c, b_{1}, \cdots, b_{N}\right)$ and let $\tau$ approach $\tau_{c}$ to find

$$
\tilde{v}_{0}=c \Lambda\left(\tilde{v}_{0}\right), \quad \text { for } \quad \tau=\tau_{c} .
$$

The function $C(z)$ can be canceled as a constant of proportionality. This shows that Eq. (4.38) contains both the criticality condition and the neutron source function $\tilde{v}_{0}$ for a critical reactor.

For the spectral analysis of $\Lambda$ for a given $b_{1}, \cdots, b_{N}$ and $\tau$, one determines all values of $c$ for which (4.49) holds. An equivalent analysis which is easier computationally is to fix $c$ and $b_{1}, \cdots, b_{N}$ and search for all $\tau_{i}>\tau_{c}$ such that

$\operatorname{det} \mathbf{M}\left(\tau_{i}, c, b_{1}, \cdots, b_{N}\right)=0 \quad i=1, \cdots$.

This defines the condition for existence of higherorder even eigenfunctions of $\Lambda$ for each of the $\tau_{i}$. Again, by the ordering (4.41) of the eigenvalues of $\mathbf{M}$, the corresponding eigenfunction is given by $v_{0}\left(x, \mu, \tau_{i}\right)$. By a trivial modification of the above results, we may obtain the equations for $Q(x, \mu, z)$ which would then lead to a different set of $\tau_{i}$ for which odd eigenfunctions of $\Lambda$ exist.

Finally, we note that the above results should yield a method for solving the homogeneous Milne problem for $c>1$. That is, if for any subsequence $\left(i_{i}\right)$ the limit exists in

$$
\rho(x, \mu)=\lim _{i \rightarrow \infty} v_{0}\left(x, \mu, \tau_{i j}\right),
$$

then $\rho$ solves the equation

$$
\rho=c \Lambda(\rho), \quad \tau=\infty,
$$

and, hence, is the solution to the Milne problem. Further discussion of this procedure appears in the next section.

We will now apply the results of this section to specific cases, namely linear anisotropic scattering and isotropic scattering.

\section{EXAMPLES IN SLAB GEOMETRY}

\section{A. Linear Anisotropic Scattering}

In this section we will study the criticality problem assuming that the scattering function is given by

$$
f\left(\mu, \mu^{\prime}\right)=c\left[1+b_{1} \mu \mu^{\prime}\right] .
$$

Demanding positivity of $f$ leads easily to the following inequality for $b_{1}$ :

$$
\left|b_{1}\right|<1 \text {. }
$$

Using the results of Sec. 3, we find that an elementary calculation for $k$ and $\gamma$ gives

$$
\begin{aligned}
& k(\sigma, \nu)=c\left[1-(c-1) b_{1} \sigma \nu\right], \\
\gamma(\sigma)= & \frac{1}{2} k(\sigma, \sigma) \\
= & \frac{1}{2}(c-1) c b_{1}\left[1 /(c-1) b_{1}-\sigma^{2}\right] .
\end{aligned}
$$

The above equation immediately reveals the zeros of $\gamma$ as

$$
\pm \zeta_{0}= \pm\left[(c-1) b_{1}\right]^{-\frac{1}{2}} .
$$

The function $\lambda$ may be calculated from (3.7). The result is

$$
\begin{array}{r}
\lambda(z)=1-c\left[z-(c-1) b_{1} z^{3}\right] \tanh ^{-1}(1 / z) \\
-(c-1) c b_{1} z^{2} .
\end{array}
$$

It is easily verified that, if either $c<1+1 / b_{1}$ and $1>b_{1}>0$, or $-1<b_{1} \leq 0$, then $\lambda$ has only one pair of zeros, $\pm z_{0}$, in the complex plane cut from -1 to 1 . They are pure imaginary for $c>1$ and real for $c<1$. In this case, $\alpha=1$ and $\alpha+\beta=2$ so that two arbitrary constants $a_{0}, a_{1}$ arise. By combining (4.30) and (4.36) Eqs. (4.32) for $a_{0}$ and $a_{1}$ may be written as follows:

$$
\mathbf{M}\left(\tau, c, b_{1}, \cdots, b_{N}\right) \mathrm{a}=\mathbf{v}(x, \mu, \tau),
$$

where

$$
\begin{array}{r}
M_{11}=z_{0} E\left(z_{0}\right)-z_{0} e^{-\tau / z_{0}} E\left(-z_{0}\right)-L\left(g_{0}\right)\left(z_{0}\right) \\
-e^{-\tau / z_{0}} L\left(g_{0}\right)\left(-z_{0}\right), \\
M_{12}=z_{0}^{2} E\left(z_{0}\right)+z_{0}^{2} e^{-\tau / z_{0}} E\left(-z_{0}\right)-L\left(g_{1}\right)\left(z_{0}\right) \\
-e^{-\tau / z_{0}} L\left(g_{1}\right)\left(-z_{0}\right), \\
M_{21}=\zeta_{0} E\left(\zeta_{0}\right)+\zeta_{0} E\left(-\zeta_{0}\right)+e^{\tau / \zeta 0} L\left(g_{0}\right)\left(\zeta_{0}\right) \\
-e^{-\tau / \zeta_{0}} L\left(g_{0}\right)\left(-\zeta_{0}\right), \\
M_{22}=\zeta_{0}^{2} E\left(\zeta_{0}\right)-\zeta_{0}^{2} E\left(-\zeta_{0}\right)+e^{\tau / \zeta_{0}} L\left(g_{1}\right)\left(\zeta_{0}\right) \\
-e^{-\tau / \zeta_{0}} L\left(g_{1}\right)\left(-\zeta_{0}\right),
\end{array}
$$


and

$$
\begin{aligned}
v_{1}=l_{1}\left(x, \mu, z_{0}\right)- & L(\tilde{g})\left(x, \mu, z_{0}\right) \\
& -e^{-\tau / z_{0}} L(\tilde{g})\left(x, \mu,-z_{0}\right), \\
v_{2}=-e^{\tau / \zeta_{0}} L(\tilde{g})\left(x, \mu, \zeta_{0}\right) & \\
& +e^{-\tau / \zeta_{0}} L(\tilde{g})\left(x, \mu,-\zeta_{0}\right) .
\end{aligned}
$$

The functions $\tilde{g}, g_{0}$, and $g_{1}$ are computed from (4.22). The quantities $L\left(g_{i}\right)$ and $L(\tilde{g})$ are then computed by (4.21).

To find the critical thickness, we let $\tau$ increase, with $c$ and $b_{1}$ fixed, until

$$
\operatorname{det} \mathbf{M}\left(\tau_{c}, c, b_{1}\right)=\mathbf{0} .
$$

From (4.50) the corresponding critical source function is given by

$J(x, \mu)=M_{21} v_{1}\left(x, \mu, \tau_{c}\right)-M_{11} v_{2}\left(x, \mu, \tau_{c}\right)$.

Since the determinant of $M$ depends on the functions $\tilde{g}, g_{1}$, and $g_{0}$, the solution of (5.9) for $\tau_{c}$ implies a search for $\tau_{c}$ by repeated calculations of the $g_{0}, g_{1}$ functions for various values of $\tau$.

Many physically interesting cases are in the range $c-1 \ll 1$. In this case, $\tau_{c}$ is large and approaches infinity as $c$ decreases to 1 . Then from (4.22) we approximate $g_{0}$ and $g_{1}$ by 0 to obtain an approximation for $\tau_{c}$ from (5.9), as

$$
\begin{aligned}
& {\left[1-\frac{z_{0}}{\zeta_{0}} \frac{E\left(\zeta_{0}\right)+E\left(-\zeta_{0}\right)}{E\left(\zeta_{0}\right)-E\left(-\zeta_{0}\right)}\right] E\left(z_{0}\right)} \\
& \quad=\left[1+\frac{z_{0}}{\zeta_{0}} \frac{E\left(\zeta_{0}\right)+E\left(-\zeta_{0}\right)}{E\left(\zeta_{0}\right)-E\left(-\zeta_{0}\right)}\right] E\left(-z_{0}\right) \exp \left(-\tau_{0} / z_{0}\right) .
\end{aligned}
$$

The flux, which is derived from the source function $J$ by integration, can be shown to reduce to

$$
\begin{aligned}
\rho\left(x, \tau_{c}\right) \equiv \int_{-1}^{1} J\left(x, \mu, \tau_{c}\right) d \mu \\
=A \cos \left(\frac{\tau_{c}-2 x}{2\left|z_{0}\right|}\right),
\end{aligned}
$$

for some constant $A$.

The quantities appearing in (5.11) can be related to the solution of the Milne problem. For fixed $c$ and $b_{1}$, we readily see that there is a sequence $\left\{\tau_{i}\right\}$ tending to infinity for which

$$
\operatorname{det} \mathbf{M}\left(\tau_{i}, c, b_{1}\right)=\mathbf{0} .
$$

Also we conclude from (5.7) that, even though $z_{0}$ is pure imaginary,

$$
\begin{aligned}
& \lim _{i \rightarrow \infty} \exp \left(-\tau_{i} / z_{0}\right)=\frac{E\left(z_{0}\right)}{E\left(-z_{0}\right)} \\
& \times \frac{\zeta_{0}\left[E\left(\zeta_{0}\right)-E\left(-\zeta_{0}\right)\right]-z_{0}\left[E\left(\zeta_{0}\right)+E\left(-\zeta_{0}\right)\right]}{\zeta_{0}\left[E\left(\zeta_{0}\right)-E\left(-\zeta_{0}\right)\right]+z_{0}\left[E\left(\zeta_{0}\right)+E\left(-\zeta_{0}\right)\right]}
\end{aligned}
$$

For each such $\tau_{i}$, we have an eigenfunction $J\left(x, \mu, \tau_{i}\right)$ of $\Lambda$ given by (5.10) with $\tau_{c}$ replaced by $\tau_{i}$. We see then that

$$
\begin{aligned}
\lim _{i \rightarrow \infty} & J\left(x, \mu, \tau_{i}\right)=\lim _{i \rightarrow \infty}\left[\zeta_{0} E\left(\zeta_{0}\right)+\zeta_{0} E\left(-\zeta_{0}\right)\right] \\
& \times\left[k\left(\mu, z_{0}\right) \exp (-x / z)+k\left(-\mu, z_{0}\right)\right. \\
& \left.\times \exp \left[\left(x-\tau_{i}\right) / z_{0}\right]\right]+O[\exp (-x)]
\end{aligned}
$$

This function, part of which we have merely abbreviated by $O[\exp (-x)]$, gives a solution to the Milne problem.

Defining $\rho_{i}$ by

$$
\rho_{i}(x)=\int_{-1}^{1} J\left(x, \mu, \tau_{i}\right) d \mu
$$

we see that

$$
\begin{array}{r}
\lim _{i \rightarrow \infty} \rho_{i}(x)=A\left[\exp \left(-x / z_{0}\right)+\exp \left(x / z_{0}\right)\right. \\
\left.\times \lim _{i \rightarrow \infty} \exp \left(-\tau_{i} / z_{0}\right)\right]+O[\exp (-x)] .
\end{array}
$$

If we neglect the term $O[\exp (-x)]$, we see that the asymptotic part of (5.17) leads naturally to the concept of an extrapolation distance $\tau_{a}$, at which the asymptotic solution vanishes. Then from (5.17) and (5.14), we can relate $\tau_{a}$ and $\tau_{c}$ by

$$
\exp \left(-\tau_{c} / z_{0}\right)=-\exp \left(2 \tau_{a} / z_{0}\right),
$$

or

$$
\tau_{c}=\pi\left|z_{0}\right|-2 \tau_{a} .
$$

Higher-order approximations to the criticality condition may be achieved by iterating the Fredholm equations (4.22) and taking these iterates into account in the determinant of $\mathbf{M}$.

If $c>1+1 / b_{1}$ and $1>b_{1}>0$, the function $\lambda$ has two pairs of zeros in the cut plane. ${ }^{3}$ Both pairs are pure imaginary if $c b_{1}>3$, and one pair is imaginary and the other is real if $c b_{1}<3$. This extra pair of zeros increases $\alpha$ to two and increases the size of the matrix $\mathbf{M}$ to three by three. The additional required matrix elements may be easily found. Since in these cases $c$ is restricted by $c>2$, the critical dimension will not be large.

In the case $c b_{1}<3$, it is again possible to find a solution to the Milne problem as the limit of eigenfunctions for a sequence of values of $\tau$. However, in the case $c b_{1}>3$ it is not clear that this can be done. The trouble is due to the fact that the determinant of $\mathbf{M}$ as well as the eigenfunctions contain two oscillatory functions of $\tau$, exp $\left(-\tau / z_{0}\right)$ and $\exp \left(-\tau / z_{1}\right)$ for pure imaginary numbers $z_{0}$ and $z_{1}$, 
and it is not clear that the $J\left(x, \mu, \tau_{i}\right)$ converge as the $\tau_{i}$ tend to infinity.

\section{B. Isotropic Scattering}

We now specialize to the case where

$$
f\left(\mu, \mu^{\prime}\right)=c
$$

The functions $k, \gamma$, and $\lambda$ are now simply given by

$$
\begin{aligned}
& k(\sigma, \nu)=c, \quad \gamma(\sigma)=\frac{1}{2} c, \\
& \lambda(z)=1-c z \tanh ^{-1}(1 / z) .
\end{aligned}
$$

The matrix $\mathbf{M}$ degenerates into a single element and we find that

$$
a_{0}=\frac{l_{1}\left(x, z_{0}\right)-L(\tilde{g})\left(x, z_{0}\right)-\exp \left(-\tau / z_{0}\right) L(\tilde{g})\left(x,-z_{0}\right)}{z_{0} E\left(z_{0}\right)-z_{0} \exp \left(-\tau / z_{0}\right) E\left(-z_{0}\right)-L\left(g_{0}\right)\left(z_{0}\right)-\exp \left(-\tau / z_{0}\right) L\left(g_{0}\right)\left(-z_{0}\right)} .
$$

We can immediately write down the criticality condition $^{14}$

$$
\begin{aligned}
z_{0} E\left(z_{0}\right)- & z_{0} e^{-\tau_{c} / z_{0}} E\left(-z_{0}\right)-L\left(g_{0}\right)\left(z_{0}\right) \\
& -\exp \left(-\tau_{c} / z_{0}\right) L\left(g_{0}\right)\left(-z_{0}\right)=0,
\end{aligned}
$$

and the flux

$$
\begin{aligned}
\rho(x)=l_{1}\left(x, z_{0}\right) & -L(\tilde{g})\left(x, z_{0}\right) \\
& -\exp \left(-\tau / z_{0}\right) L(\tilde{g})\left(x,-z_{0}\right) .
\end{aligned}
$$

As before, the ratio $E\left(z_{0}\right) / E\left(-z_{0}\right)$ may be related to the extrapolation distance for the Milne problem. If this is done we may rewrite the criticality condition (5.23) as

$$
\begin{gathered}
\frac{1+\frac{c}{2} \int_{0}^{1} \frac{g_{0}(\sigma) d \sigma}{\lambda^{-}(\sigma) E^{+}(\sigma)\left(\sigma+z_{0}\right)}}{1+\frac{c}{2} \int_{0}^{1} \frac{g_{0}(\sigma) d \sigma}{\lambda^{-}(\sigma) E^{+}(\sigma)\left(\sigma+z_{0}\right)}} \\
=-\exp \left[-\left(\tau+2 \tau_{a}\right) / z_{0}\right] .
\end{gathered}
$$

Since both sides of this equation are complex numbers on the unit circle, we may equate arguments to get

$$
\begin{aligned}
& \tan ^{-1}\left[\frac{-\left|z_{0}\right| \frac{c}{2} \int_{0}^{1} \frac{g_{0}(\sigma) d \sigma}{\lambda^{-}(\sigma) E^{+}(\sigma)\left(\sigma^{2}+\left|z_{0}\right|^{2}\right)}}{1+\frac{c}{2} \int_{0}^{1} \overline{\lambda^{-}(\sigma) E^{+}(\sigma)\left(\sigma^{2}+\left|z_{0}\right|^{2}\right)}}\right] \\
& =\frac{1}{2}\left[\pi-\frac{\left(\tau+2 \tau_{a}\right)}{\left|z_{0}\right|}\right] \text {. }
\end{aligned}
$$

From Sec. 4, the function $g_{0}$ is the solution to the following Fredholm integral equation:

$$
\begin{aligned}
g_{0}(\nu)= & -\nu \exp (-\tau / \nu) E(-\nu) \\
& \times\left[1-\frac{c}{2} \int_{0}^{1} \frac{g_{0}(\sigma) d \sigma}{\lambda^{-}(\sigma) E^{+}(\sigma)(\sigma+\nu)}\right] .
\end{aligned}
$$

Since $\gamma$ is positive $\left(=\frac{1}{2} c\right)$, we see by the results ${ }^{14}$ A. Leonard, and T. W. Mullikin, The RAND Cor-
poration, RM-3256-PR (1962). of Sec. 4 that the following iterative scheme for approximating $g_{0}$ will converge uniformly to the solution for any $c$ and $\tau$ :

$$
\begin{gathered}
g_{0}^{(0)}(\nu)=0 \\
g_{0}^{(n+1)}(\nu)=-\nu \exp (-\tau / \nu) E(-\nu) \\
\times\left[1-\frac{c}{2} \int_{0}^{1} \frac{g_{0}^{(n)}(\sigma) d \sigma}{\lambda^{-}(\sigma) E^{+}(\sigma)(\sigma+\nu)}\right] .
\end{gathered}
$$

Hence, using the above precedure, the critical slab thickness for the entire range of values of $c(1<c<\infty)$ may be determined. An analogous iteration scheme may be written for $\tilde{g}(x, \nu)$.

Mitsis $^{4}$ has also formulated the slab criticality problem for isotropic scattering using Case's singular eigenfunction expansion. As expected, his resulting Fredholm kernel is adjoint to the kernel in Eq. (5.27). Corresponding iterations of the two adjoint equations, however, give precisely the same results for the neutron flux and critical thickness.

\section{SPHERICAL REACTOR: ISOTROPIC SCATTERING}

The neutron density $\rho_{0}(x)$, in a homogeneous critical sphere with isotropic fission production and scattering, satisfies the equation

$$
\begin{aligned}
x \rho_{0}(x)= & \frac{c}{2} \int_{-\tau / 2}^{+\tau / 2} y \rho_{0}(y) \\
& \times\left[\int_{0}^{1} \frac{\exp (-|x-y| / \mu)}{\mu} d \mu\right] d y,
\end{aligned}
$$

where $\tau$ is the sphere diameter. ${ }^{1}$ With the substitution

$$
\rho(x)=\left(x-\frac{1}{2} \tau\right) \rho_{0}\left(x-\frac{1}{2} \tau\right),
$$

Eq. (6.1) becomes identical to the integral equation for the neutron density in a slab reactor of thickness $\tau$. However, from (6.2) we have the following symmetry condition on $\rho$ :

$$
\rho(x)=-\rho(\tau-x) .
$$

We require therefore the first odd eigenfunction of $\Lambda$. 
According to the discussion in Sec. 4, the odd eigenfunctions are found by considering Eq. (4.1) for $Q$. By a few simple sign changes in the analysis of Sec. $5 \mathrm{~B}$, we find that the constant $a_{0}$ is given by

$$
a_{0}=\frac{-l_{2}\left(x, z_{0}\right)+\exp \left(-\tau / z_{0}\right) L(\tilde{g})\left(x,-z_{0}\right)-L(\tilde{g})\left(x, z_{0}\right)}{z_{0} \exp \left(-\tau / z_{0}\right) E\left(-z_{0}\right)+z_{0} E\left(z_{0}\right)+L\left(g_{0}\right)\left(z_{0}\right)-\exp \left(-\tau / z_{0}\right) L\left(g_{0}\right)\left(-z_{0}\right)},
$$

with the functions $\tilde{g}$ and $\tilde{g}_{0}$ now given by the solution to the following Fredholm equations:

$$
\begin{gathered}
\tilde{g}(x, \nu)=l_{2}(x, \nu)+L(\tilde{g})(x, \nu), \\
g_{0}(\nu)=\nu \exp (-\tau / \nu) E(-\nu)+L\left(g_{0}\right)(\nu) .
\end{gathered}
$$

Equation (6.4) leads to the following sphere criticality condition ${ }^{14}$ :

$\tan ^{-1}\left[\frac{\left|z_{0}\right| \frac{c}{2} \int_{0}^{1} \frac{g_{0}(\sigma) d \sigma}{\lambda^{-}(\sigma) E^{+}(\sigma)\left(\sigma^{2}+\left|z_{0}\right|^{2}\right)}}{1+\frac{c}{2} \int_{0}^{1} \frac{\sigma g_{0}(\sigma) d \sigma}{\lambda^{-}(\sigma) E^{+}(\sigma)\left(\sigma^{2}+\left|z_{0}\right|^{2}\right)}}\right]$

$$
=\left[\frac{\tau+2 \tau_{a}}{2\left|z_{0}\right|}-\pi\right] \text {. }
$$

Again iterative schemes completely analogous to (5.26) may be defined which converge uniformly to the solution of (6.5) and (6.6) for every $c$ and $\tau$.

In the criticality condition for the sphere (6.7) and the slab (5.26), the multiple-valued function "arctan" appears. The multiple roots of these equations, $\tau_{i}$, correspond to the $\tau_{i}$ of (4.53) and, therefore, define the existence of higher-order eigenfunctions of the operator $\Lambda$ for isotropic scattering.

\title{
Some Exact Radial Integrals for Dirac-Coulomb Functions*
}

\author{
J. T. Reynolds, D. S. Onley, And L. C. Biedenharn \\ Duke University, Durham, North Carolina
}

(Received 14 August 1963)

\begin{abstract}
The zero energy loss Dirac-Coulomb integrals are evaluated using the technique of contour integration. The expressions obtained have a closed analytic form, showing that these integrals are formally similar to the corresponding classical and nonrelativistic quantum mechanical, zero energy loss integrals which also have exact elementary solutions.

Application of the zero energy loss Dirac-Coulomb integrals occurs in inelastic electron scattering and similar problems. The investigation of the finite energy loss Dirac-Coulomb integrals requires a study of the zero energy loss integrals as a preliminary.
\end{abstract}

\section{INTRODUCTION}

B $Y$ using the technique of multipole field expansion, the treatment of problems involving energy transfer from charged particles in the Coulomb field, in cases for which retardation effects are negligible, can often be reduced essentially to the evaluation of integrals containing, in the integrand, factors of the form, $r^{-L-1} Y_{L}^{M}$. Such is the case, for example, in the processes of Coulomb excitation, bremsstrahlung, pair production, etc. The evaluation of these integrals is not a completely straightforward task since the integrals with which we are concerned are more general than the Sonine-Schafheitlin integral ${ }^{\mathbf{1}}$ and

* Supported in part by the Army Research Office (Durham), and the National Science Foundation.

1 W. Magnus and F. Oberhettinger, Special Functions of Mathematical Physics (Chelsea Publishing Company, New York, 1949), p. 35. in a similar way display discontinuous behavior in the zero energy loss limit.

The treatment of such integrals in the classical ${ }^{2-5}$ and nonrelativistic quantum mechanical ${ }^{2,3,6,7}$ cases has been discussed in several places. The authors were led to the problem of extending this work to the relativistic case during the course of calculating, accurately, the differential cross section for

${ }^{2}$ L. C. Biedenharn and P. J. Brussaard, Coulomb Excitation (to be published by Oxford University Press).

${ }^{3} \mathrm{~K}$. Alder, A. Bohr, T. Huus, B. Mottelson, and A. Winther, Rev. Mod. Phys. 28, 432 (1956).

$4 \mathrm{~K}$. Alder and A. Winther, Kgl. Danske Videnskab. Selskab, Mat. Fys. Medd. 31, No. 1 (1956).

5 L. D. Landau and E. M. Lifschitz, The Classical Theory of Fields (Pergamon Press, Ltd., London, 1959).

6. C. Biedenharn, J. L: McHale, and R. M. Thaler, Phys. Rev. 100, 376 (1955).

${ }_{7}$ M. Goldstein, R. M. Thaler, and L. C. Biedenharn, Los Alamos Scientific Laboratory Rept. LA-2106 (1957) (unpublished). 